\title{
CYTOKERATIN-18 FRAGMENTS FOR PREDICTING STEATOHEPATITIS IN PATIENTS WITH NONALCOHOLIC FATTY LIVER DISEASE
}

\author{
Babak O., Zhelezniakova N., Molodan V., Prosolenko K., Lapshyna K., Vizir M. \\ Kharkiv National Medical University, Department of Internal Medicine No.1, \\ Kharkiv, Ukraine
}

https://doi.org/10.35339/ic.6.3.137-142

\begin{abstract}
The aim of the study was to investigate metabolic parameters, cytokeratin 18 (CK-18) levels in blood plasma and the state of the liver parenchyma of patients with non-alcoholic fatty liver disease (NAFLD) in non-alcoholic steatohepatitis (NASH) stage. Materials and methods: Fasting serum CK-18 was measured in 30 NASH patients (mean age (49.3 \pm 3.6 ). The control group consisted of 20 practically healthy volunteers. The diagnosis of NADH was established on the basis of clinical, biochemical and instrumental data. In addition to basic laboratory tests and ultrasonographic examination, the Fibromax test (Biopredective, France) was performed. Results: In patients with NAFLD, the lipid profile showed a tendency to hyperand dyslipidemia. The obtained data showed a significant increase in the parameters of enzymatic activity of the liver, the average level of aspartate aminotransferase (AST) exceeded the control group 2.5 times, the alanine aminotransferase (ALT) - 2.9 times, respectively $(<0.05)$. In NAFLD in NASH stage CK-18 levels in blood plasma were significantly increased, exceeded the results of the control group 3 and more times, and allowed to establish NASH in patients of the main group. Conclusions: Increased levels of CK-18 in NASH patients as well as its association with atherogenic dyslipidemia and increasing of liver enzymatic activity were revealed. The obtained data showed the possibility of using biomarker for non invasive diagnostics of NASH and represent the prospect of further research.

Key words: nonalcoholic fatty liver disease, nonalcoholic steatohepatitis, noninvasive diagnosis, cytokeratin-18.
\end{abstract}

\section{Introduction}

The prevalence of non-alcoholic fatty liver disease (NAFLD) around the world is approximately $30 \%$. However, the true prevalence of NAFLDs and associated diseases is unknown, mainly due to the lack of reliable and widely used diagnostic tests. NAFLD is traditionally considered as a consequence of metabolic syndrome (MetS). However, the link between the components of MetS and NAFLD, in particular type 2 diabetes mellitus (T2DM), hypertension (HTN) and cardiovascular disease (CVD), is more complicated than previously thought. NAFLD-associated diseases affect various systems and have different pathogenetic pathways with a large number of clinical evidence,

Corresponding Author:

Kateryna Lapshyna, MD, PhD, Assistant Professor of the Department of Internal medicine No. 1, Kharkiv National Medical University, Ukraine. E-mail: ekaterinalapshyna@gmail.com indicate that NAFLD can precede and promote the development of T2DM, HTN, atherosclerosis and CVD. The risk of developing these cardiometabolic diseases is directly related to the severity of NAFLD. Existing data suggest that the presence and severity of NAFLD are associated with an increased risk of T2DM and HTN. Moreover, long-term prospective studies indicate that the presence and severity of NAFLD are independently associated with episodes of lethal and non-fatal cardiovascular events [4, $10,11]$.

Fatty liver can be diagnosed using ultrasound, while liver biopsy remains the gold standard for the evaluation of fibrosis and NASH. Although other non-invasive tests have been proposed, they still need to be tested in large multicenter studies. Since steatosis, NAFLD and NASH and associated metabolic diseases are an economic burden, the search for inexpensive NAFLD diagnostic methods and NASH directly is a priority $[2,7,9]$. 
The invasiveness, the complexity of the procedure and the mistakes in the interpretation of the biopsy require development of new safe and accurate methods of NAFLD diagnosis. Therefore, there is a growing need for noninvasive diagnosis using biomarkers. Previous studies have shown that some serum biomarkers have the potential for NAFLD and NASH diagnosis.

Povsic, M. et al. (2018), in their meta-analysis of all the biomarkers for NAFLD diagnosis, concluded that cytokeratin 18 (CK18) is a marker of hepatocyte apoptosis and is the most widely studied biomarker of steatohepatitis [12].

CK-18 has been widely studied since 2009 and has showen its high specificity and sensitivity both for NAFLD and NASH in many studies. In recent years, studies of CK-18 in NAFLD patients have been combined with other markers and scales, and aimed at increasing of diagnostic value of NASH and liver fibrosis progression. Thus, Juan Pablo Arab (2018) et al. in their research analyzed CK-18 levels in 41 patients of the Chilean population with a biopsy-proven NAFLD and showed that CK-18 levels were significantly higher in patients with NASH than those in patients without NASH (183.6 U/L [97.4-734.4] vs. 117.2 $\mathrm{U} / \mathrm{L}$ [83.8-954.8], $\mathrm{p}=0.016$ ). The level of CK18 was a reliable predictor of NASH with 0.732 AUC (95\% CI, 0.572-0.897). The CK-18 cut-off of $130.5 \mathrm{U} / \mathrm{L}$ had a sensitivity of $92.9 \%$, a specificity of $63 \%$, a positive predictive value of $56.5 \%$, and a negative predictive value of $94.4 \%$. Scientists concluded that CK-18 is a reliable noninvasive marker for NASH and can be used to predict both NASH and liver fibrosis in patients with NAFLD, CK-18 and NAFLD score [1].

Darweesh, Samar K. et al. (2019) studied CK-18 diagnostic value in combination with FibroScan and showed improved accuracy of diagnosis and strong direct link of the marker with NAFLD progression [6].

Toshifumi Tada et al. (2018) in their research with 130 patients histologically verified NASH, created a new scoring system, FIC-22, to predict NASH using CK18 levels and the FIB-4 index. The areas under the ROC (AUROC) FIC-22 curve and the NAFIC score estimation were 0.82 (95\% CI, 0.75-0.89) and 0.71 (95\% CI, 0.62$0.78)(\mathrm{p}=0.044)$. In addition, AUROC FIC-22 score for predicting the presence of fibrosis $(\mathrm{F} \geq$ 1) was $0.78(95 \% \mathrm{CI}, 0.70-0.85)$. It indicates a high predictive accuracy of the FIC-22 scale for patients with NAFLD not only for steatohepatitis, but also for the presence of liver fibrosis [14].
Therefore, the study of the possibility of using non-invasive biomarkers for the diagnosis of liver fibrosis and the progression of NASH in conjunction with HTN is a promising area of research.

\section{Purposes, subjects and methods:}

2.1. Purpose of the study was to investigate metabolic parameters, cytokeratin 18 (CK-18) levels in blood plasma and the state of the liver parenchyma of patients with NAFLD in the stage of NASH.

\subsection{Subjects \& Methods}

30 patients with NAFLD were examined, aged 30 to 60 years (mean age $(49.3 \pm 3.6)$ years). The control group consisted of 20 practically healthy volunteers. The gender distribution of patients admitted to the study was presented by $56.6 \%(17 / 30)$ of males and $43.3 \%(13 / 30)$ of females. Statistically significant differences in the gender distribution of patient groups were not obtained. Clinical laboratory parameters of blood, urine and biochemical parameters of blood were determined in all patients. The diagnosis of NAFLD was established in accordance with the criteria outlined in the clinical protocol of the European Association of Gastroenterologists, in common with the Association for the Study of Diabetes and the Association for the Study of Obesity [8]. The blood plasma levels of CK-18 fragments were carried out using the enzyme immunoassay method with the ELISA kit. The condition of liver parenchyma was studied using biochemical test Fibrotest (Biopredictive). An echosonographic examination of the abdominal cavity was performed after fasting using an ultrasound scanner of the expert class "Vivid-3" ("General Electric", USA).

Exclusion criteria were diffuse connective tissue diseases, oncological diseases, acute inflammatory diseases, history of viral hepatitis, toxic (alcohol), drug-induced, congenital metabolic diseases of the liver.

Statistical processing of the results was carried out using "Excel 2010" (Microsoft) and SPSS 19 software packages. Continuous variables are represented as medians and values of $25^{\text {th }}$ and $75^{\text {th }}$ percentiles - Me (Q1-Q3). Student's criterion (t-criterion) was used in order to determine the statistically significant difference in continuous variables in two independent groups for dependent and independent samples, and nonparametric Mann-Whitney and Wilcoxon criteria were used for the distribution of the investigated characteristic, different from the normal one. The difference was considered to be reliable at the 
bilateral level of significance $\mathrm{p}<0.05$ for all conducted analyzes.

\section{Conflict of interests}

The authors declare that they have no competing interests.

\section{Results and discussion}

Given the typical metabolic profile of patients with NASH, determination of anthropometric parameters, lipid and carbohydrate profiles is of particular interest. The average BMI of the patients in the main group corresponded to the classes of people with increased weight, in particular $30 \%$ of patients $(n=6)$ were overweight and $30 \%(n=6)$ had obesity of the 1st degree (Table 1).
Comparison of indicators of functional state of the liver and markers of its inflammation in patients with NASH and control group is presented in Table. 2.

The obtained data showed the increased enzymatic activity of the liver in patients with NAFLD. Alanine transaminase (ALT) was 2.9 times, aspartate transaminase (AST) -2.5 times and gamma-glutamyltransferase (GGT) -2.8 times higher compared to those in practically healthy individuals. The inflammatory liver markers and total bilirubin did not exceed the generally accepted normal values, but significantly differed between the groups. Thus, the median of alkaline phosphatase in the main group was 1910 units,

Table 1

Anthropometric indices of patients in the main group and control group

\begin{tabular}{|c|c|c|c|}
\hline Index & $\begin{array}{c}\text { NAFLD group } \\
(\mathrm{n}=30)\end{array}$ & $\begin{array}{c}\text { Control group } \\
(\mathrm{n}=20)\end{array}$ & $\mathrm{p}$ \\
\hline $\mathrm{BMI}, \mathrm{kg} / \mathrm{m} 2$ & $26.9(24.8 ; 28.9)$ & $25.35(24.3 ; 27.6)$ & \\
\hline WHR & $1.02(0.98 ; 1.06)$ & $0.89(0.87 ; 0.91)$ & $<0.001$ \\
\hline
\end{tabular}

Note: ${ }^{*} \mathrm{p}<0.05$ - the difference between the values of group's indicators is statistically significant.

The waist to hip ratio (WHR) in NAFLD patients demonstrated the presence of abdominal obesity in comparison with the control group, in which this index exceeded the normal value in $10 \%(\mathrm{n}=2)$ cases of overweight. the thymol test was $2.1 \mathrm{U}$ versus 1205 units and $1.4 \mathrm{U}$ in the control group, respectively $(\mathrm{p}<0.001)$.

It is well known that the presence of NAFLD is associated with hyperlipidemia, metabolic syndrome and obesity. In recent years, evidence

Indicators of the functional state of the liver, lipid and carbohydrate profiles

Table 2 in patients of the main group and control group

\begin{tabular}{|c|c|c|c|}
\hline Index & $\begin{array}{c}\text { NAFLD group } \\
(n=30)\end{array}$ & $\begin{array}{c}\text { Control group } \\
(n=20)\end{array}$ & $p$ \\
\hline ALT, U/I & $72.0(60.7 ; 84.5)$ & $\begin{array}{c}24.5 \\
(20.2 ; 29.2) \\
\end{array}$ & 0.03 \\
\hline AST, U/l & $57.5(48.5 ; 66.7)$ & $\begin{array}{c}22.5 \\
(17.0 ; 27.7)\end{array}$ & 0.007 \\
\hline GGT, U/I & $\begin{array}{c}63.0 \\
(59.7 ; 70.5) \\
\end{array}$ & $\begin{array}{c}22.5 \\
(18.0 ; 26.7)\end{array}$ & 0.002 \\
\hline Alkaline phosphatase & $\begin{array}{c}1910 \\
(1745 ; 2127)\end{array}$ & $\begin{array}{c}1205 \\
(990 ; 1432)\end{array}$ & $<0.05$ \\
\hline Thymol test, U & $\begin{array}{c}2.1 \\
(1.7 ; 3.5)\end{array}$ & $\begin{array}{c}1.4 \\
(0.74 ; 2.0)\end{array}$ & $<0.05$ \\
\hline Total bilirubin, mkmol/l & $\begin{array}{c}14.2 \\
(12.4 ; 17.2)\end{array}$ & $\begin{array}{c}9.1 \\
(8.4 ; 9.3) \\
\end{array}$ & $<0.05$ \\
\hline Total cholestirol, $\mathrm{mmol} / \mathrm{l}$ & $5.4(5.0 ; 6.2)$ & $4.3(3.7 ; 4.7)$ & 0.001 \\
\hline $\mathrm{TG}, \mathrm{mmol} / \mathrm{l}$ & $1.55(1.3 ; 1.75)$ & $0.8(0.6 ; 1.0)$ & 0.001 \\
\hline $\mathrm{HDL}, \mathrm{mmol} / \mathrm{l}$ & $3.3(3.0 ; 3.9)$ & $2.3(1.8 ; 2.7)$ & 0.001 \\
\hline Aterogenic ratio & $0.7(0.60 ; 0.80)$ & $0.4(0.3 ; 0.52)$ & 0.001 \\
\hline LDL, $\mathrm{mmol} / \mathrm{l}$ & $0.59(0.38 ; 0.8)$ & $1.38(1.2 ; 1.5)$ & 0.001 \\
\hline $\mathrm{TC}, \mathrm{mmol} / \mathrm{l}$ & $3.1(2.2 ; 4.0)$ & $2.04(1.5 ; 2.5)$ & 0.001 \\
\hline Glucose, mmol/l & $\begin{array}{c}5.4 \\
(5.1 ; 5.6)\end{array}$ & $\begin{array}{c}4.47 \\
(4.1 ; 4.97)\end{array}$ & 0.001 \\
\hline Insulin, U/I & $22.7(21.2 ; 25.4)$ & $\begin{array}{c}13.4 \\
(11.6 ; 17.1)\end{array}$ & 0.002 \\
\hline HOMA-IR & $5.47(5.0 ; 6.03)$ & $2.7(2.1 ; 3.4)$ & 0.001 \\
\hline
\end{tabular}


based data claimed that lipid metabolism disorders could be present in NAFLD patients without obesity [17]. Comparison of the lipid profile of patients in the main group and control group showed a significant increase in the parameters of total cholesterol, triglycerides (TG), LDLcholesterol and lowering of antiatherogenic HDLcholesterol $(p<0.001)$. The atherogenic ratio of the main group 1.5 times exceeded that of healthy volunteers $(p<0.001)$. Changes in all parameters of lipid profile can be explained by the presence of NASH and overweight in examined patients.

The state of carbohydrate metabolism in NASH patients characterized by hyperinsulinemia compared with the control group, and as a consequence, insulin resistance. All indications were significantly different in comparison between the groups, but an increase in fasting glucose level was not higher than normal values, and the levels of insulin and the HOMA-index in 1.5 and 2 times exceeded the results obtained in the control group, respectively. In patients involved in the study, there were stages of liver fibrosis, which on the international scale Metavir correspond to the values F0, F1, F2-3. Stage F4 of fibrosis was not detected. The distribution of the stages of liver fibrosis among patients in the comparison group found that the overwhelming number of patients had the stages F1 and F2-3, namely $80 \%$. According to the results of the analysis, the total sample of patients was distributed as follows: 0 stage of fibrosis was detected in $20 \%$ of patients with NAFLD, F1 in $30 \%$ and $\mathrm{F} 2-3$ in $50 \%$ of patients (Fig. 1).
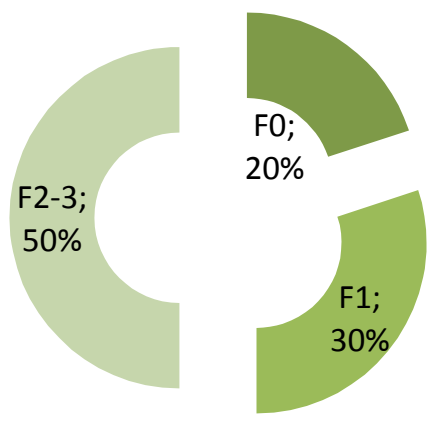

Fig. 1. Distribution of stages of liver fibrosis in NASH patients

Analysis of the enzymatic activity of the liver indicated its growth with an increase in the stage of fibrosis. Thus, the values of AST and ALT were significantly higher in patients with F2-3 compared to those at the F0 stage of fibrosis, that was also confirmed in comparison of the groups $\mathrm{F} 1$ and $\mathrm{F} 2-3(\mathrm{p} 2<0.05, \mathrm{p} 3<0.05)$, whereas comparing the results of patients with the stage $\mathrm{F} 0$ of fibrosis with the $\mathrm{F} 1$ group revealed only a tendency to increase the activity of the inflammatory process in the liver. The obtained results and their association with the stage of fibrosis by such indicators as alkaline phosphatase, thymol test and total bilirubin were non-specific and no significant differences were found.

Similar results were obtained in evaluation of the lipid profile, where the HDL cholesterol index increased with liver fibrosis stage in comparing groups, except comparison of the groups with stages F0 and F2-3. Also, the increase in the rates in comparison of the F1 and F2-3 groups was determined by the level of platelets, which were significantly higher with fibrosis growth $(p<0.05)$. According to the carbohydrate profile, there was no significant difference between the groups.

Determination of the level of cytokine CK-18 in the blood plasma of patients with NAFLD showed its increase. It is noteworthy that the levels of CK-18 in 3 and more times exceeded those of practically healthy individuals.

Discussion. Related data was obtained and published by Li Xue et al. (2018) in the histological study of liver tissue in rats, levels of CK-18 increased with progression of liver disease [15]. Feldstein et al. in 2008 at first found the properties of circulating fragments of CK-18 to reflect NASH in patients with NASH. Since then, intensive biomarker studies have been ongoing, indicating a high diagnostic value of CK-18 and its link with NASH [16].

Cusi K. et al. (2014) in their study found that levels of CK-18 in the blood plasma of patients with NAFLD increased with steatosis, inflammation and liver fibrosis stages growth reflecting the severity of the disease. CK-18 AUROC for predicting NAFLD, NASH, or fibrosis was $0.77(95 \% \mathrm{CI}=0.71-0.84), 0.65$ $(95 \% \mathrm{CI}=0.59-0.71)$, and $0.68(95 \% \mathrm{CI}=0.61-$ 0.75 ) respectively. Overall sensitivity/specificity for NAFLD, NASH and fibrosis was 63\% (57$70 \%) / 83 \%$ (69-92\%), 58\% (51-65\%)/68\% (59-76\%) and 54\% (44-63\%) / 85\% (75-92\%) respectively [5].

Speliotes E. et al. in the study of paired liver biopsies, found a correlation between the level of CK-18 fragments in blood plasma and NASH in patients with NAFLD [13]. Chalasani N. et al. studied the cohort of NAFLD patients: levels of CK-18 fragments in plasma $\geq 225 \mathrm{U} / \mathrm{L}, \geq 250 \mathrm{U} /$ 1 or $\geq 300 \mathrm{U} / 1$ had sensitivity of $70 \%, 60 \%$, or $53 \%$, respectively; Specificity $82 \%, 93 \%$, or $100 \%$; 


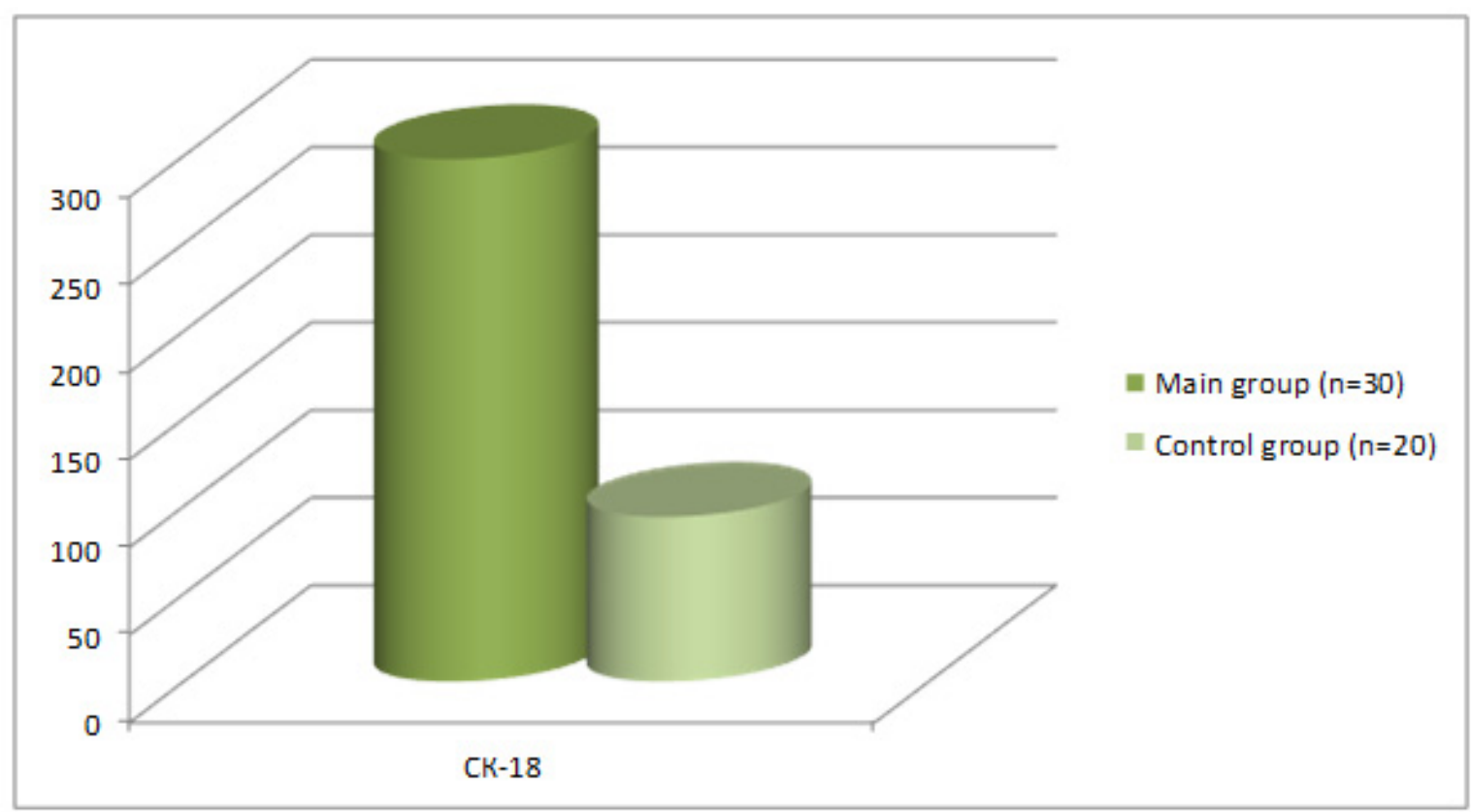

Fig. 2. Levels of CK-18 in patients of the main group and control group

Note: $\mathrm{p}<0.02-$ statistically significant differences in comparison with control group indicators.

positive predictive value $84 \%, 95 \%$, and $100 \%$; and a negative predictive value of $73 \%, 69 \%$, and $67 \%$ for the diagnosis of NASH, respectively [3]. It makes possible using of CK-18 fragments for NAFLD activity determination and treatment response.

\section{Conclusions}

The metabolic profile of NAFLD patients with $\mathrm{NASH}$ is characterized by an increase in enzymatic activity of the liver, elevated cholesterol and its fractions and hyperinsulinemia. The levels of CK-18 in the blood plasma of the examined patients were significantly higher than those in the control group, and were associated with metabolic state disorders.

The CK-18 is a promising predictive marker and can be used in NASH diagnosis. Further evidence is needed to improve our understanding of NASH diagnostic, especially as fibrosis stages advance.

\section{References}

1. Arab, J. P., Arrese, M., \& Trauner, M. (2018). Recent insights into the pathogenesis of nonalcoholic fatty liver disease. Annual Review of Pathology: Mechanisms of Disease, 13, 321-350.

2. Araujo, A. R., Rosso, N., Bedogni, G., Tiribelli, C., \& Bellentani, S. (2018). Global epidemiology of non?alcoholic fatty liver disease/non?alcoholic steatohepatitis: What we need in the future. Liver International, 38, 47-51.

3. Chalasani, Naga, et al. "Genome-wide association study identifies variants associated with histologic features of nonalcoholic Fatty liver disease." Gastroenterology 139.5 (2010): 1567-1576.

4. Chesnokova, L.V. (2015). Clinical and pathogenetic significance of non-alcoholic fatty liver disease in the formation of high cardiovascular risk in patients with metabolic syndrome. Possibilities of correction: Diss.

5. Cusi, K., Chang, Z., Harrison, S., Lomonaco, R., Bril, F., Orsak, B., ... \& Louden, C. (2014). Limited value of plasma cytokeratin-18 as a biomarker for NASH and fibrosis in patients with nonalcoholic fatty liver disease. Journal of hepatology, 60(1), 167-174.

6. Darweesh, S. K., AbdElAziz, R. A., Abd-ElFatah, D. S., AbdElazim, N. A., Fathi, S. A., Attia, D., \& AbdAllah, M. (2019). Serum cytokeratin-18 and its relation to liver fibrosis and steatosis diagnosed by FibroScan and controlled attenuation parameter in nonalcoholic fatty liver disease and hepatitis $\mathrm{C}$ virus patients. European journal of gastroenterology \& hepatology, 31(5), 633-641.

7. de Alwis, N. M. W., \& Day, C. P. (2008). Non-alcoholic fatty liver disease: the mist gradually clears. Journal of hepatology, 48, S104-S112. 
8. European Association for the Study of the Liver, \& European Association for the Study of Diabetes (EASD. (2016). EASL-EASD-EASO Clinical Practice Guidelines for the management of non-alcoholic fatty liver disease. Obesity facts, 9(2), 65-90.

9. Ferraioli, G., Tinelli, C., Dal Bello, B., Zicchetti, M., Filice, G., Filice, C., \& Liver Fibrosis Study Group. (2012). Accuracy of reatime shear wave elastography for assessing liver fibrosis in chronic hepatitis C: a pilot study. Hepatology, 56(6), 2125-2133.

10. Lokhov, P. G., Maslov, D. L., Balashova, E. E., Trifonova, O. P., Medvedeva, N. V., Torkhovskaya, T. I., ... \& Shestakova, E. A. (2015). Mass spectrometry analysis of blood plasma lipidome as a method for diagnosing diseases, evaluating the effectiveness and optimizing drug therapy. Biomedical Chemistry, 61 (1), 7-18.

11. Lonardo, A., Nascimbeni, F., Mantovani, A., \& Targher, G. (2018). Hypertension, diabetes, atherosclerosis and NASH: cause or consequence? Journal of hepatology, 68 (2), 335-352.

12. Povsic, M., Wong, O. Y., Perry, R., \& Bottomley, J. (2019). A Structured Literature Review of the Epidemiology and Disease Burden of Non-Alcoholic Steatohepatitis (NASH). Advances in therapy, $1-21$.

13. Speliotes, Elizabeth K., et al. "Genome-wide association analysis identifies variants associated with nonalcoholic fatty liver disease that have distinct effects on metabolic traits." PLoS genetics 7.3 (2011): e1001324.

14. Tada, T., Kumada, T., Toyoda, H., Saibara, T., Ono, M., \& Kage, M. (2018). New scoring system combining the FIB-4 index and cytokeratin-18 fragments for predicting steatohepatitis and liver fibrosis in patients with nonalcoholic fatty liver disease. Biomarkers, 23(4), 328-334.

15. Xue, L., Lu, X., He, J., Zhang, T., Wu, X., Zhang, Y., ... \& Geng, Y. (2018). Serum CK 18-M30 reflect liver pathological severity during NAFLD progression in a rat model. Pathology-Research and Practice, 214(11), 1778-1786.

16. Younossi, Z. M., Loomba, R., Anstee, Q. M., Rinella, M. E., Bugianesi, E., Marchesini, G., ... $\&$ Ratziu, V. (2018). Diagnostic modalities for nonalcoholic fatty liver disease, nonalcoholic steatohepatitis, and associated fibrosis. Hepatology, 68(1), 349-360.

17. Yousef, M. H., Al Juboori, A., Albarrak, A. A., Ibdah, J. A., \& Tahan, V. (2017). Fatty liver without a large "belly": Magnified review of non-alcoholic fatty liver disease in non-obese patients. World journal of gastrointestinal pathophysiology, 8(3), 100.

Received: 21-May-2019

Accepted: 09-Sep-2019 